\title{
ABSOLUTELY CONTINUOUS AND LOCALLY QUASI-INVARIANT MEASURES ON LOCALLY COMPACT SEMIGROUPS
}

\author{
HENRY A. M. DZINOTYIWEYI
}

\begin{abstract}
Let $S$ be a locally compact semigroup and $T$ a locally compact subsemigroup of $S$. It is shown that, for a large class of semigroups $S$, if $T$ is 'reasonably large', the absolutely continuous measures on $T$ extend to measures whose translates by some points of $S$ are absolutely continuous on $S$. As a consequence we prove the absolute continuity of translates of some measures related to a locally quasi-invariant measure.
\end{abstract}

1. Introduction. Throughout this paper, let $S$ denote a locally compact jointly continuous topological semigroup, $M(S)$ the set of all bounded complex-valued Radon measures on $S$ and $C_{0}(S)$ the set of all continuous functions on $S$ which vanish at infinity. Identifying $M(S)$ as the first dual of $C_{0}(S)$, it is well known that $M(S)$ is a Banach algebra under the usual norm, \|\| , and convolution multiplication given by

$$
\mu * \nu(f)=\iint f(x y) d \mu(x) d \nu(x) \quad\left(\mu, \nu \in M(S) ; f \in C_{0}(S)\right) .
$$

In the papers [1], [2], [3] and [7] certain subspaces of the algebra $M(S)$ are studied-the purpose being to find an analogue of $L^{1}(G)$ for a locally compact group $G$. Let $M_{a}^{l}(S)\left(M_{a}^{r}(S)\right)$ be the set of all measures $\mu$ in $M(S)$ such that the mapping $x \rightarrow \bar{x} *|\mu|(x \rightarrow|\mu| * \bar{x})$ of $S$ into $M(S)$ is weakly continuous, where $\bar{x}$ denotes the point mass at $x$ and $|\mu|$ the total variation of $\mu$. Then taking $M_{a}(S)$ to be $M_{a}^{l}(S) \cap M_{a}^{r}(S)$ we have the set studied in [2], [3] and [9]. In particular, from [2, Lemma 2.4 and Theorem 3.2] and [3, Theorem 2.6] we note that $M_{a}(S)$ is an $L$-ideal of $M(S)$ (i.e. $M_{a}(S)$ is a norm closed linear subspace of $M(S)$ such that for any $\nu, \mu \in M(S)$ we have (i) if $\nu \ll|\mu|$ and $\mu \in M_{a}(S)$ then $\nu \in M_{a}(S)$ and (ii) if $\nu \in M_{a}(S)$ then $\nu * \mu, \mu * \nu \in$ $M_{a}(S)$ ). Further, when $S$ is a group then $M_{a}(S)$ (or $\left.M_{a}^{l}(S)\right)$ can be identified with the usual group algebra $L^{1}(S)$ (see e.g. [6, 19.27 and 20.31]). A different approach is made in [7], where it is shown that if $m$ is a positive measure on $S$ such that $m\left(x^{-1} E\right)=m\left(E x^{-1}\right)=0$ whenever $m(E)=0$ for all Borel $E \subset S$ and $x \in S$, then taking $L(S, m)=\{\nu \in M(S): \nu \ll m\}, L(S, m)$ has some

Received by the editors April 25, 1977 and, in revised form, August 11, 1977.

AMS (MOS) subject classifications (1970). Primary 43A05, 43A20; Secondary 43A35, 43A60.

Key words and phrases. Absolutely continuous measure, locally quasi-invariant measure, translate of a measure, foundation semigroup. 
properties related to those of the usual group algebra $L^{1}(G)$ of a locally compact group $G$. It is natural to ask whether there is any relationship between $L(S, m)$ and $M_{a}(S)$. We will give a partial answer to this problem in Theorem 3.7. In $\$ 4$ we will give an example of a semigroup $S$ equal to the support of a measure $m$ with the property (studied in [7] and) mentioned above but with $M_{a}(S)$ equal to zero.

Further, it is of interest to know the relationship between $M_{a}(T)$ and $M_{a}(S)$, when $T$ is a locally compact subsemigroup of $S$. For example for those subsemigroups $T$ of some semigroups $S$ for which we are able to show that $M_{a}(T)=\left\{\nu \in M_{a}(S):|\nu|(S \backslash T)=0\right\}$ (cf. Corollary 3.4) we have for instance: If $S$ is commutative and equal to the carrier space of $M_{a}(S)$, then the bounded multiplicative linear functionals on $\left\{\nu \in M_{a}(S):|\nu|(S \backslash T)=\right.$ $0\}$ are in one-to-one correspondence with the set of all continuous semicharacters on $T$. This follows easily from [3, Theorem 4.4] and one can easily note that [8, Theorem 13] is a special case of this result.

However, when $S$ is not cancellative such subsemigroups $T$ for which $M_{a}(T)=\left\{\nu \in M_{a}(S):|\nu|(S \backslash T)=0\right\}$ are not easy to come by (see e.g. Remark 3.6). In that case we obtain a generalization of such an identification in Theorem 3.2.

We begin by collecting together some results and introducing further notation, in $\S 2$, which will be needed in $\$ \S 3$ and 4 .

2. Preliminaries. For any sets $A, B \subseteq S$ and $x \in S$, let $A B=\{x y: x \in$ $A, y \in B\}, A^{-1} B=\{y \in S: a y \in B$ for some $a \in A\}, x^{-1} B=\{x\}^{-1} B$, $A^{-1} x=A^{-1}\{x\}$ and similarly define $A B^{-1}, B x^{-1}$ and $x A^{-1}$. We say a measure $\mu$ is absolutely continuous if $\mu \in M_{a}(S)$. The measure $\bar{x} * \mu$ is the left translate of $\mu$ by $x \in S(\mu \in M(S))$. For any regular Borel measure $m$ on $S$, let $L_{m}=\left\{x \in S:|m|(B)=0\right.$ implies $m\left(x^{-1} B\right)=0$ for all Borel $\left.B \subset S\right\}$, $\operatorname{supp}(m)=\{x \in S:|m|(X)>0$ whenever $X$ is an open neighbourhood of $x\}$ and if $E \subset S$ is a Borel set we define the measure $m_{\mid E}$ by $m_{\mid E}(B)=m(B \cap$ $E$ ) for all Borel $B \subseteq S$. For $\mathcal{Q} \subseteq M(S)$ and Borel $E \subseteq S$ we let $\mathcal{C}_{1 E}=\{\mu \in$ $Q:|\mu|(S \backslash E)=0\}$. For any $E \subseteq S$, let $d(E)=\left\{x \in S: x \in \operatorname{supp}\left(\mu_{\mid B}\right)\right.$ for some Borel set $B \subset E$ and $\left.\mu \in M_{a}(S)\right\}$.

Following [2] and [3] we will say $S$ is a foundation semigroup if $d(S)=S$, and following [4] we will say a regular Borel measure $m$ is (left) locally quasi-invariant if $d\left(L_{m}\right) \neq \varnothing$.

Whenever $S$ has an identity element $1, S_{1}$ will denote the set of all $x \in S$ such that $X^{-1} x \cap x X^{-1}$ is a neighbourhood of 1 , whenever $X$ is a neighbourhood of $x$. This set $S_{1}$ plays a very significant role whenever $S$ is a foundation semigroup with an identity element 1 (see e.g. [4] and [9]). In particular, from $[9,6.9$ and 7.8$]$ we have the following:

2.1 LEMMA. If $S$ is a foundation semigroup with an identity element 1 , then $S_{1}$ is an ideal of $S$ (i.e. $x y, y x \in S_{1}$ for all $x \in S_{1}$ and $y \in S$ ) and $d\left(S \backslash S_{1}\right)=0$ (i.e. $M_{a}(S)$ is 'concentrated' on $S_{1}$ ). 
The next result was proved by us in [4].

2.2 Proposition. Let $S$ be a foundation semigroup with identity element 1 and $\mu \in M(S)$. Then

(i) $\bar{x} * \mu \in M_{a}(S)$, for all $x \in S_{1}\left(d\left(L_{\mu}\right) \cap L_{\mu}\right)$;

(ii) if $F$ is a subsemigroup containing a countable subset $O_{\mu}$ such that if $\bar{x} *|\mu|(B)=0\left(x \in O_{\mu}\right)$ then $\bar{x} *|\mu|(B)=0(x \in F)$, we have $\bar{x} *|\mu| \in$ $M_{a}(S)\left(x \in S_{1}(d(F) \cap F) F\right)$.

We will need the next lemma in Theorem 3.2.

2.3 LEMMA. If $T$ is a locally compact subsemigroup of $S$, there is an isometric algebra isomorphism $\theta: M(S)_{\mid T} \rightarrow M(T)$ given by $\theta(\mu)(B)=\mu(B \cap T)$ for all Borel $B \subseteq T$ and $\mu \in M(S)_{\mid T}$.

Proof. Since $T$ is a locally compact subset of $S, T$ is the intersection of an open subset and a closed subset of $S$ (see e.g. [11, 18.4]). So $T$ is a Borel subset of $S$. It is not hard to observe that the proof of [12, Theorem 3.4] contains the proof of our lemma.

Finally for any $E \subset S$, let $M_{a}^{l}(S, E)=\left\{\mu \in M(S): \bar{x} *|\mu| \in M_{a}(S)\right.$ for some $x \in E\}$ and note that $M_{a}^{l}(S, E)$ is a subalgebra of $M(S)$ such that if $\nu \in M(S), \mu \in M_{a}^{l}(S, E)$ and $\nu \ll|\mu|$ then $\nu \in M_{a}^{l}(S, E)$. (This follows from the fact that $M_{a}(S)$ is an $L$-ideal as pointed out in the introduction.) Further if $S$ is a group and $E \neq \varnothing$, then evidently $M_{a}^{l}(S, E)=M_{a}(S)$.

3. Main results. Throughout this section $S$ is a foundation semigroup with an identity element 1 and $T$ is a locally compact subsemigroup of $S$. We will only give proofs for the left-handed case, from which the right-handed and two-sided cases should be clear.

3.1 LEMMA. Let $E$ be a subset of $S$ with $d(E)$ nonempty and $\mu \in M(S)$. If $\left\{\bar{x} *|\mu|: x \in E^{n}\right\}$ is a relatively weak compact subset of $M(S)(n \in \omega)$ and $F=\bigcup{ }_{n=1}^{\infty} E^{n}$, then

$$
\bar{x} *|\mu| \in M_{a}(S) \quad\left(x \in S_{1}(d(F) \cap F) F\right) .
$$

Here $E^{n+1}=E E^{n}(n \in \omega)$ and $E^{1}=E$.

Proof. By [5, Théorème 2], the relative weak compactness of $\{\bar{x} *|\mu|$ : $\left.x \in E^{n}\right\}$ implies that $\left\{\bar{x} *|\mu|: x \in E^{n}\right\}$ satisfies item (b) of the theorem of [10]. From [10, pp. 139 and 140] it follows that there is a countable set $A_{n} \subset E^{n}$ such that $\bar{x} *|\mu|(B)=0\left(x \in A_{n}\right)$ implies $\bar{x} *|\mu|(B)=0\left(x \in E^{n}\right)$.

Let $O_{\mu}=\cup_{n=1}^{\infty} A_{n}$ and observe that $\bar{x} *|\mu|(B)=0\left(x \in O_{\mu}\right)$ implies $\bar{x} *|\mu|(B)=0(x \in F)$. By Proposition 2.2(ii) we are done.

3.2 THEOREM. Suppose $d(T) \neq \varnothing$. Then $M_{a}^{l}\left(T, T \cap S_{1}\right)$ is isometrically algebra isomorphic to

$$
M_{a}^{l}\left(S, T \cap S_{1}\right)_{\mid T}=\left\{\nu \in M_{a}^{l}\left(S, T \cap S_{1}\right):|\nu|(S \backslash T)=0\right\} .
$$

Proof. Let $\theta$ be as stated in Lemma 2.3. Let $\nu \in M_{a}^{l}\left(S, S_{1} \cap T\right)_{\mid T}$. There 
is $x \in T \cap S_{1}$ with $\bar{x} *|\nu| \in M_{a}(S)$. By [2, 2.4 and 3.2], $(\bar{x} *|\nu|)_{\mid T} \in M_{a}(S)$. Consequently $\theta\left((\bar{x} *|\nu|)_{\mid T}\right) \in M_{a}(T)$. Since $x \in T$, by the definition of $\theta$, we get

$$
\theta\left((\bar{x} *|\nu|)_{\mid T}\right)=\theta(\bar{x} *|\nu|)=\bar{x} * \theta(|\nu|)=\bar{x} *|\theta(\nu)| .
$$

Thus $\theta(\nu) \in M_{a}^{l}\left(T, T \cap S_{1}\right)$.

On the other hand suppose $\nu^{\prime} \in M_{a}^{l}\left(T, T \cap S_{1}\right)$. There is a unique $\nu$ in $M(S)_{\mid T}$, such that $\theta(\nu)=\nu^{\prime}$, by Lemma 2.3. There is $x_{0} \in T \cap S_{1}$ such that $\bar{x}_{0} *\left|\nu^{\prime}\right| \in M_{a}(T)$. Since $d(T) \neq \varnothing$, there is a measure $\eta \in M_{a}(S)$ such that $\eta(T)>0$. By the regularity of $\eta$, we can find a compact subset $E$ contained in $T$, such that $\eta(E)>0$. Thus $d(E) \neq \varnothing$. Observe that $E^{n}$ is a compact subset of $T(n \in \omega)$. So, by our definition of $M_{a}(T),\left\{\bar{y} *\left(\bar{x}_{0} *\left|\nu^{\prime}\right|\right): y \in E^{n}\right\}$ is a weakly compact subset of $M(T)(n \in \omega)$. For all $x, y \in T$ we have

$$
\bar{y} *\left(\bar{x} *\left|\nu^{\prime}\right|\right)=\bar{y} * \bar{x} * \theta(|\nu|)=\theta(\bar{y} *(\bar{x} *|\nu|)) \text {. }
$$

It follows that $\left\{\left(\bar{y} *\left(\bar{x}_{0} *|\nu|\right)\right)_{\mid T}: y \in E^{n}\right\}$ is a weakly compact subset of $M(T)$ and hence of $M(S)$, by Hahn-Banach Theorem. But since $|\nu|(S \backslash T)$ $=0$, we have

$$
\begin{aligned}
\left(\bar{y} *\left(\bar{x}_{0} *|\nu|\right)\right)_{\mid T} & =\left(\overline{y x}_{0} *|\nu|\right)_{\mid T}(B) \\
& =\left(\overline{y x}_{0} *|\nu|\right)(B \cap T)+|\nu|(S \backslash T) \\
& =\overline{y x}_{0} *|\nu|(B \cap T)+\overline{y x}_{0} *|\nu|(S \backslash T) \\
& =\overline{y x}_{0} *|\nu|(B) \\
& =\bar{y}^{*}\left(\bar{x}_{0} *|\nu|\right)(B),
\end{aligned}
$$

for every Borel $B \subset S$ and $y \in E^{n}$. It follows that $\left\{\bar{y}_{*}\left(\bar{x}_{0} *|\nu|\right): y \in E^{n}\right\}$ is a weakly compact subset of $M(S)(n \in \omega)$. By Lemma 3.1 we then get

$$
\bar{x} *|\nu| \in M_{a}(S) \quad\left(x \in S_{1}(d(F) \cap F) F x_{0}\right),
$$

where $F=\cup{ }_{n=1}^{\infty} E^{n}$. Since $d\left(S \backslash S_{1}\right)=\varnothing$ and $d(T) \neq \varnothing$, we have $S_{1} \cap T$ $\neq \varnothing$. Since $T$ is a subsemigroup and $S_{1}$ is an ideal of $S$,

$$
\left(S_{1} \cap T\right)(d(F) \cap F) F x_{0} \subseteq T \cap S_{1} .
$$

Consequently $\nu \in M_{a}^{l}\left(S, T \cap S_{1}\right)$.

It is now evident from Lemma 2.3 that the restriction of $\theta$ to $M_{a}^{l}(S, T \cap$ $\left.S_{1}\right)_{\mid T}$ is the required isometric algebra isomorphism.

3.3 CoROLlaRY. If $d(T) \neq \varnothing$, then $M_{a}(T) \subseteq M_{a}^{l}\left(S, S_{1}\right)$.

3.4 Corollary. Suppose $S$ is concellative (i.e. $x y=x z$ or $y x=z x$ implies $y=z(x, y, z \in S))$ and $d(T) \neq \varnothing$. Then

$$
M_{a}(T)=\left\{\nu \in M_{a}(S):|\nu|(S \backslash T)=0\right\} .
$$

Proof. Evidently $M_{a}(S)_{\mid T} \subseteq M_{a}(T)$ so we only need to show that $M_{a}(T)$ $\subseteq M_{a}(S)_{\mid T}$. Let $\nu \in M_{a}(T), K$ a compact subset of $S$ and suppose $x_{\alpha} \rightarrow x$ in $S$. By Theorem 3.2, there is $x_{0} \in S_{1}$ with $\bar{x}_{0} *|\nu| \in M_{a}(S)$. Hence 


$$
\begin{aligned}
|| \nu\left|\left(K x_{\alpha}^{-1}\right)-\right| \nu\left|\left(K x^{-1}\right)\right| & =|| \nu\left|\left(x_{0}^{-1}\left(x_{0} K\right) x_{\alpha}^{-1}\right)-\right| \nu\left|\left(x_{0}^{-1}\left(x_{0} K\right)\right) x^{-1}\right| \\
& \text { by cancellation } \\
& =\left|\bar{x}_{0} *\right| \nu\left|\left(\left(x_{0} K\right) x_{\alpha}^{-1}\right)-\bar{x}_{0} *\right| \nu\left|\left(\left(x_{0} K\right) x^{-1}\right)\right| \rightarrow 0 .
\end{aligned}
$$

This implies that $\nu \in M_{a}^{r}(S)$, by [2, Lemma 2.4]. Similarly $\nu \in M_{a}^{l}(S)$ and hence $\nu \in M_{a}(S)_{\mid T}$.

In particular, we have the following improvement of [1, Proposition 1.16(iii)]:

3.5 COROLlaRY. Let $S$ be a group with a Haar measure $m$. If $m(T) \neq 0$, then $M_{a}(T)=\{\nu \in M(S): \nu \ll m$ and $|\nu|(S \backslash T)=0\}$ (cf. [9, Theorem 4.11]).

Proof. This follows from Corollary 3.4 and the fact that $M_{a}(S)=L(S, m)$ (see [6, 19.27 and 20.31]).

3.6 REMARK. In general no identification of $M_{a}(T)$ and $M_{a}(S)_{\mid T}$ can be achieved even when $S$ is compact commutative and $d(T)=T$.

For example consider $S=[0,1]$ with the usual topology and the operation $x y=\min (x+y, 1)$. This example is borrowed from [9]. $S$ is evidently a foundation semigroup. Take $T=\left[\frac{1}{2}, 1\right]$ and observe that $M_{a}(T)=M(T)$. However, for all $x \in\left[\frac{1}{2}, 1\right), \bar{x} \notin M_{a}(S)$.

As an application of Theorem 3.2, we obtain the following theorem which relates local quasi-invariance to absolute continuity.

3.7 THEOREM. Let $m$ be a left locally quasi-invariant positive regular Borel measure on $S$, then $L(S, m) \subseteq M_{a}^{l}\left(S, S_{1}\right)$. (Here $m$ is not necessarily bounded.)

Proof. Let $\nu \in L(S, m)$ be fixed. We start by observing that if $E \subset S$ is such that $d(E)=E$ and $E$ is compact, then

$$
d\left(E^{n}\right)=E^{n} \quad(n \in \omega) .
$$

It is sufficient to prove (1) for the case where $n=2$, and an easy induction type of argument will yield the general case. Recalling that $M_{a}(S)$ is norm closed in $M(S)$ and $\mu \ll \eta$ with $\eta \in M_{a}(S)$ implies $\mu \in M_{a}(S)$ (see [2, 3.2 and 2.4]), it is evident that if $B \subset S$, then $d(B)$ is closed and every point of $d(B)$ is a cluster point of $B$. In particular since $E^{2}$ is compact we then get $d\left(E^{2}\right) \subseteq E^{2}$. Now if $x, y \in E$ and $V$ is any Borel neighbourhood of $x y$, there is a compact neighbourhood $X$ of $x$ with $x y \in X y \subset V$. Since $x \in d(E)$, there is positive measure $\mu \in M_{a}(S)$ with $\mu(X \cap E)>0$. Now $\mu * \bar{y} \in$ $M_{a}(S)[2,2.4$ and 3.6], and

$\mu * \bar{y}\left(V \cap E^{2}\right) \geqslant \mu * \bar{y}(X y \cap E y) \geqslant \mu\left(X y y^{-1} \cap E y y^{-1}\right) \geqslant \mu(X \cap E)>0$. Hence $x y \in d\left(E^{2}\right)$. So the equality $d\left(E^{2}\right)=E^{2}$ follows.

We now construct $T$ such that $T \subseteq d(T), \nu \in M(T), d\left(L_{m}\right) \cap T \neq \varnothing, 1$ is an interior point of $T$ and $T$ is $\sigma$-compact.

By the regularity of $\nu$ and the local compactness of $S$, we can choose a sequence of compact sets $\left\{C_{n}\right\} \subset S$ such that each $C_{n}$ is the closure of an 
open set (and so $\left.d\left(C_{n}\right)=C_{n}\right), C_{n} \subset C_{n+1}$ and $\left\|\nu-\nu_{\mid C_{n}}\right\|<1 / n$ ( $n \in \omega$ ). Thus $\|\nu\|=|\nu|\left(\cup_{n=1}^{\infty} C_{n}\right)$. Let $D$ be a compact set which is the closure of an open neighbourhood of the identity element 1 . Since $d\left(L_{m}\right) \neq \varnothing$ and clearly $d\left(L_{m} \backslash d\left(L_{m}\right)\right)=\varnothing$, there is a compact set $F \subset L_{m}$ such that $d(F) \neq \varnothing$. Taking $K_{n}=C_{n} \cup D \cup d(F)$, we observe that $K_{n}$ is compact and $d\left(K_{n}\right)=$ $K_{n}(n \in \omega)$. So by $(1), d\left(\left(K_{n}\right)^{k}\right)=\left(K_{n}\right)^{k}(n, k \in \omega)$. Taking

$$
T=\bigcup_{\substack{1<k<\infty \\ 1<n<\infty}}\left(K_{n}\right)^{k},
$$

it is evident that $T$ is as stated above when we identify $\nu$ with $\nu_{\mid \cup_{n-1}^{\infty},} C_{n}$.

Since $M_{a}(S)_{\mid T} \subseteq M_{a}(T)$ and $T \subseteq d(T), T$ is thus a foundation semigroup with identity element 1 . Let $T_{1}$ be defined with respect to $T$ in a way $S_{1}$ was defined with respect to $S$ and observe that $T_{1} \subseteq S_{1}$. We define the measure $m_{T}$ on $T$ by

$$
m_{T}=\sum_{\substack{1<k<\infty \\ 1<n<\infty}} 2^{-(n+k)} \frac{m_{\mid\left(K_{n}\right)^{k}}}{1+m\left(\left(K_{n}\right)^{k}\right)} .
$$

Then $m_{T} \in M(T)$. Suppose $m_{T}(B)=0$ for some Borel $B \subset T$. Then $m(B \cap$ $T$ ) $=0$ (of course Borel subsets of $T$ are also Borel subsets of $S$ since $T$ is). Since $d(F) \subset L_{m} \cap T$, we then get

$$
m\left(x^{-1} B \cap T\right) \leqslant m\left(x^{-1}(B \cap T)\right)=0 \quad(x \in d(F)) .
$$

So $m_{\mid\left(K_{k}\right)^{k}}\left(x^{-1} B\right)=0(n, k \in \omega$ and $x \in d(F))$. Hence $m_{T}\left(x^{-1} B\right)=0(x \in$ $d(F))$. If we define $d^{\prime}$ with respect to $T$ as we defined $d$ with respect to $S$, we get $d^{\prime}\left(L_{m_{T}}\right) \supseteq d(F)$. So $d^{\prime}\left(L_{m_{T}}\right) \neq \varnothing$. By Proposition 2.2(i), we then have

$$
\bar{x} * m_{T} \in M_{a}(T) \quad\left(x \in T_{1}\left(d^{\prime}\left(L_{m_{T}}\right) \cap L_{m_{T}}\right)\right) \text {. }
$$

Clearly $\bar{x} *|\nu| \ll \bar{x} * m_{T}(x \in T)$, where $\nu$ is identified with $\nu_{\mid T}$. Consequently $\bar{x} *|\nu| \in M_{a}(T)\left(x \in T_{1}\left(d^{\prime}\left(L_{m_{T}}\right) \cap L_{m_{T}}\right)\right)$. Since $T_{1}$ is an ideal of $T$ and $T_{1} \subset S_{1}$, we have proved that $\bar{x} *|\nu| \in M_{a}(T)$ for some $x \in T \cap S_{1}$. Thus $\nu \in M_{a}^{l}\left(T, T \cap S_{1}\right)$. By Theorem 3.2, it follows that $\nu \in M_{a}^{l}\left(S, S_{1}\right)$. This completes our proof.

4. An example. We construct a commutative $S$ and a Borel measure $m$ on $S$ such that $L_{m}=S$ and $\operatorname{supp}(m)=S$, but $M_{a}(S)=\{0\}$-the zero subspace of $M(S)$.

Let $S=\{0\} \cup \cup_{n=0}^{\infty}\left(\left\{n+2^{-k}: k \in \omega\right\}\right)$ with the relative topology of the line and multiplication given by $x y=\max (x, y)$ for all $x, y \in S$.

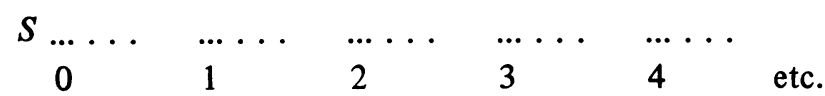

Let $\left\{x_{n}\right\}$ be an enumeration of $S$ and take $m=\sum_{n=1}^{\infty} 2^{-n} \bar{x}_{n}$. Then $L_{m}=S$ and $\operatorname{supp}(m)=S$. However $M_{a}(S)=\{0\}$. To verify the last assertion the reader can check that if $x=n+2^{-k}$, then the mapping $y \rightarrow \bar{y} * \bar{x}(\{n+2\})$ 
of $S$ into $\mathbf{R}$ is not continuous at $n+2(n, k \in \omega)$.

I am indebted to Dr. A. L. T. Paterson for useful discussions.

\section{REFERENCES}

1. A. C. Baker and J. W. Baker, Algebras of measures on a locally compact semigroup, J. London Math. Soc. 1 (1969), 249-259. MR 39 \# 7019.

2. Algebras of measures on a locally compact semigroup. II, J. London Math. Soc. 2 (1970), 651-659. MR 43 \#402.

3. Algebras of measures on a locally compact semigroup. III, J. London Math. Soc. 4 (1972), 685-695. MR 46 \#5928.

4. H. A. M. Dzinotyiweyi, Locally quasi-invariant measures, J. London Math. Soc. 15 (1977), $484-488$.

5. A. Grothendieck, Sur les applications linéaires faiblement compactes $d^{\prime}$ espaces du type $C(K)$, Canad. J. Math. 5 (1953), 129-173. MR 15, 438.

6. E. Hewitt and K. A. Ross, Abstract harmonic analysis. I, Springer-Verlag, Berlin, 1963. MR 28 \#158.

7. N. J. Rothman, An $L^{1}$-algebra for certain locally compact topological semigroups, Pacific J. Math. 23 (1967), 143-151. MR 35 \#5955.

8. N. J. Rothman and M. W. Schuh, Laplace transforms on vanishing algebras, Semigroup Forum 8 (1974), 189-214. MR 51 \#5853.

9. G. L. G. Sleijpen, Convolution measure algebras on semigroups, Thesis, Katholieke Universiteit, Toernooiveld, Nijmegen, 1976.

10. J. D. Stein, Uniform absolute continuity in spaces of set functions, Proc. Amer. Math. Soc. 51 (1975), 137-140.

11. S. Willard, General topology, Addison-Wesley, Reading, Mass. 1970. MR 41 \#9173.

12. J. C. S. Wong, Absolutely continuous measures on locally compact semigroups, Canad. Math. Bull. 18 (1975), 127-132. MR 52 \# 1168.

Department of Mathematics, The University, Aberdeen, Scotland

Current address: Mathematisch Instituut der Katholieke Universiteit, Toernooiveld, Nijmegen, The Netherlands 